Noname manuscript No.

(will be inserted by the editor)

\title{
Degree-correlations in a bursting dynamic network model
}

Fabio Vanni · Paolo Barucca

\begin{abstract}
We propose a mathematical description of a dynamic network model in which the number of links fluctuates over time according to the degreepreferences of nodes with spontaneous degree correlations and bursty events.
\end{abstract}

Keywords temporal network - stochastic process - financial networks · heterogeneity $\cdot$ degree correlations $\cdot$ bursty dynamics

JEL codes: D85, C62, G21

Fabio Vanni

Institute of Economics, Scuola Superiore Sant'Anna Piazza Martiri della Libert, 3356127 Pisa (Italia)

Tel.: +39050883710

E-mail: fa.vanni@santannapisa.it

Paolo Barucca

Department of Banking and Finance, University of Zurich, Switzerland London Institute for Mathematical Sciences, London, United Kingdom E-mail: baruccap@gmail.com 
Noname manuscript No.

(will be inserted by the editor)

\title{
Degree-correlations in a bursting dynamic network model
}

\begin{abstract}
We propose a mathematical description of a dynamic network model in which the number of links fluctuates over time according to the degreepreferences of nodes. More specifically, we consider the minimal case of a bipartite directed network where we have two groups of nodes, each group has nodes with a given capability to bear links. One group is composed of nodes that create as many links as possible, the generators. The other group is composed of nodes which delete as many links as possible, i.e., the destroyers. We provide here a novel analytical formulation of the evolution of the system through coupled Master Equations for the two interacting populations, recovering the steady state degree distributions and a new analytic description of the transient dynamics to the equilibrium. Further, fluctuations are shown to be connected to a peak in degree correlation at a critical point of the system corresponding to equal-size populations of generators and destroyers. We investigate the nature of the neighbor connectivity and the temporal assortativity of the network, noticing that degree correlation are anomalously large at criticality and that they are not a pointwise characterization of the system in time but they emerge as an aggregate temporal property. Moreover, we examine the bursty dynamics of the network as a temporal property where the system heterogeneously evolves over time alternating between periods of low and high connectivity displaying a heavy-tailed distribution in the interevent times distributions. Finally, we introduce a generalization of the model in which intermittent states can control the velocity of the network's evolution. We will also provide examples of possible economic applications of the present network model.
\end{abstract}




\section{Introduction}

The conceptual framework of network formation can accurately describe complex systems which exhibit an intricate structure of evolving connections among the units.

The common models of temporal network formation on random graphs in the literature (Holme and Saramäki, 2012, 2013, Masuda and Lambiotte, 2016) have focused on symmetric formation dynamics, where two random nodes have a certain chance to be connected through a particular attachment probability. These generative schemes are essentially based on the idea that a node can connect to another according to simultaneous bilateral agreement represented through a pairwise linking probability like in the Erdos-Renyi rationale and generalized random graphs known as hidden-variable (fitness models) (Caldarelli et al., 2002) or with a unit-wise connection pattern like in the cases of preferential attachment models (Albert and Barabási, 2002).

Among models of temporal networks, as a novelty in the literature of temporal generative models, the activity driven model (Perra et al., 2012) represents the basis for a standard model of temporal networks. In this model, link formation is driven by the activation of single nodes (according to their attributes) and a subsequent asymmetric linking function.

Other research has stressed the importance of temporal models (Bardoscia et al., 2013, Ubaldi et al., 2016) for better representing dynamical structures which arise from non-static systems.

A different line of theoretical research addresses models of strategic network formation, which has been receiving contributions in the economic literature. The game theoretic interaction has been formalized in terms of both bilateral and unilateral models of link formation, see (Jackson, 2010, Jackson and Wolinsky, 1996) and (Goyal, 2012).

In the present work we start from a dynamic network approach with a connection procedure where the network changes as individuals add and delete links over time according to a asymmetrically link formation with nonsimultaneous procedure where a proposer node makes the attempt to create a link (according to its own preference) with another node which responds to the proposal (according to its own preference). In the simplest formulation, we first analyze a network formation where the proposal is immediately accepted. The model here studied is inherently dynamic and contributes to the growing body of research on temporal networks, which have been attracting considerable attention in recent years (Holme and Saramäki, 2013, Masuda and Lambiotte, 2016) for their ability to reproduce the complex dynamical patterns in real-world systems such as spontaneous degree correlations and bursty dynamics of the network, where the system spontaneously passes from states of high connectivity to states of low connectivity. Bursty patterns are, in fact, very commonly empirically observed in economics and financial activities (Karsai et al., 2018) and degree correlations are a fundamental feature of complex systems as the direct consequence of the interaction between heterogeneous agents (Kondor et al., 2014). 
We start from the model introduced by (Liu et al., 2013), where the system dynamically evolves according to the preferences of each agent, the final network is a simple directed bipartite graph which, despite its simplicity, introduces a deep change in the network structure and its dynamics with emergent properties that arise for certain values of simple network parameters. In particular, the extreme introverts and extroverts model (XIE) has been shown (Bassler et al., 2015) to be characterized by a critical behavior beyond the Ehrenfest classification of phase transitions: the discontinuity displays an extreme Thouless effect. In such a condition we observe both a discontinuity in the phase transition plot of the order parameter and the appearance of anomalously large fluctuations in the link dynamics: features of first-order and second-order phase transitions respectively.

Despite their recent introduction, non-simultaneous asymmetric linking mechanisms are in some cases more realistic than pairwise ones and this particular model is an attempt to represent unilateral links as alternative to pairwise random graph models, in the same way as one-sided strategic network formation has come up beside the two-sided models.

For instance, in social networks like Twitter, the decision of following another user is strictly unilateral, and the ratio between followers and followed users, i.e. out-going and in-coming links, strongly characterizes the behavior and attitude of users (Grandjean, 2016).

Another interesting evidence for the presence of different agents' attitudes which drive the dynamics of a system can be widely found in financial networks analysis (Barucca and Lillo, 2015, Fricke, 2012, Iori et al., 2008, 2007), where the various financial institutions in the interbank market can be divided into different communities related to their financial management.

In recent years, financial systems have represented an important field of application for network science, which naturally captures the linkage architecture of the financial agents and their relations. Interest has increased even more as consequence of the recent financial crisis of the years 2007-2009, and a network approach is particularly crucial for assessing financial stability, since it is possible to have models and methodological tools which could describe, detect and eventually mitigate systemic risk (Battiston et al., 2016). There are many possible approaches and perspectives that one can use when studying financial systems and addressing financial network analysis, but, mainly, research has focused on the one hand on topological structures and the dynamics of network formation, and on the other hand on the dynamic processes occurring on the network.

Here, we model network formation through a new mathematical description based on a Master Equation for the degree of the network. The solution we found provides both the transient non-equilibrium dynamics and the steady state of the degree distribution. At the same time we recover the crucial property of emergence and the behavior of a phase transition as the proportion between the two interacting groups varies (Bassler et al., 2015). We define the nature of complexity in the network model as the effect of temporal hetero- 
geneity which arises from of the introduction of a proposer/responder rationale for the link formation (Lambert and Vanni, 2018).

Moreover, we investigate the limits of such approaches and shed light on a new important feature of such networks: the emergence of strong correlations among the agents when the system is at criticality, connected with the extreme Thouless effect already shown in the XIE model. We will discuss the onset of a spontaneous covariance between the two groups, which is remarkably intense at the phase transition critical point. We will study the meaning and the nature of such phenomena from the new perspective of temporal degree-degree correlations which leads us to define a temporal aggregate measure of assortativity other the common punctual one. As an additional important property of the model, we show how dynamical network interactions have the capacity to regulate and buffer unpredictable fluctuations so producing bursty events in terms of network connectivity. In conclusion, we propose a re-elaboration of the original XIE model of (Bassler et al., 2015, Liu et al., 2013), where we use a novel analytical formulation and a more detailed network analysis with a new interpretation, in particular, in terms of the degree correlations and the bursty behavior. However, those features are also present in the original model of XIE a similar analysis would have been performed.

The paper is organized as follows: in Section 1 we introduce the network model and describe in detail its rationale and dynamics. We introduce the model dynamics in terms of a stochastic process, and analyze it through a master equation for the system according to an uncorrelated bipartite graph. In Section 2, then, using a mean field approximation, we write the rate equation for the network evolution so that it is possible to obtain a numerical solution of both the degree distribution and the phase transition plot. So, we outline the new coupled master equation dynamics (CMED) for the degreedistribution of generators and destroyers, explaining how it is possible to couple them. We present numerical results on the non-equilibrium dynamics, showing how CMED succeeds in predicting the full dynamical evolution of the degree-distributions in the model outside of criticality. In section 3, we discuss degree correlations at criticality, estimating the intensity and the nature of such correlations. We detect the presence of strong degree correlations and we define a notion of aggregated assortativity seen as cumulative assortativity over a time window. Moreover we show the intrinsically dynamical nature of the network through the study of burstiness of the events among different states of the connectivity. In section 4 , we introduce a more realistic version of the agent-driven network based on the XIE model where intermittency is present in the degree trajectories. Moreover, we discuss financial and economic applications of the model. In section 5, we discuss potential economic applications of the methodology developed in the paper both in terms of a financial agent-based model and in terms of an empirical study on international trade. Finally, in Section 6 we summarize results, stressing the need for a robust methodology to model degree correlations and their role in mixed phase transitions. 


\section{The model}

The network model is based on an intergroup dynamics where individual agents connect according to a degree target, where each agent tends to reach a preferred number of neighbors. In the simplified and extreme situation modelled here, the nodes in the network are divided between a group of link generators (with $N_{1}$ units) and a group of link destroyers (with $N_{0}$ units). The size of the graph is $N=N_{1}+N_{0}$ which is kept fixed over the network evolution.

The network formation dynamics is defined as follows:

- A single agent is randomly chosen

- If the selected node is a generator:

- its degree is always smaller then its target degree (high-target node)

- it randomly selects a non-previously connected node among the group of destroyers

- it creates a directed link with the selected node.

- If the drawn node is a destroyer:

- its degree is always larger then its target degree (low-target node)

- it selects one of its randomly chosen neighbors which belongs to the group of generators.

- it cuts the link with the selected node.

Actually, the previous prescription considers only active nodes, in the sense that if we pick a generator which is connected with every destroyer (full generator-node), the prescription jumps to a new selection of another node. The same is true if an empty destroyer is picked. The resulting network is a simple bipartite directed graph where high-target agents are only able to generate links (when possible) and the low-target agents which can only destroy links with its neighbors (if any). Neither multi-links nor intragroup links are considered. If we were to also consider the possibility that generators could create links among themselves (intra-group links) the cross-group and bipartite assumptions we used is broken. The only difference between the two approaches is the fact that by also considering intra-group links the degree distribution of generators is different from the destroyers' one by a constant factor. This difference comes from the fact that generators have a fixed number of links with each other (after a transient) which do not vary over time so do not contribute to the fluctuation analysis we discussed in the present paper. However, the bipartite structure we use, turned out to be useful for a new description and discussion of the model in terms of common and important concepts in the network literature such as degree correlations and burstiness, which are relevant also in the economics discipline.

At this level, the drawn node forces the selected neighbor to follow its attitude, we will see a generalization where there is a chance that the neighbor opposes to the proposal of the acting node. This network formation perspective is crucial to define an interesting dynamics in the connectivity fluctuations as well as in the rise of emergent properties such as correlations among units in the system. 
The creation/destruction dynamics can be described as a stochastic process along the lines of a generic birth-death process for the links dynamics. Let us define $\rho_{1}(k, t)$ (degree distribution) the probability of finding a generator with degree $k$ at time $t$, and $p_{0}^{(n)}(k, t)$ the neighbor degree distribution (Dorogovtsev and Mendes, 2013, Newman, 2010, Pastor-Satorras et al., 2001, Vázquez et al., 2002) that is the probability that a randomly chosen neighbor (generator) of a randomly chosen destroyer node has degree $k$. The bipartite structure allows us to write the master equation for the degree distribution as:

$$
\begin{aligned}
\rho_{1}(k, t+1) & =\rho_{1}(k, t)+ \\
& +\frac{N_{1}}{N} \frac{1}{N_{1}} \rho_{1}(k-1, t)-\frac{N_{1}}{N} \frac{1}{N_{1}} \rho_{1}(k, t)+ \\
& -\frac{N_{0}}{N} \frac{1}{N_{1}} \epsilon_{0} p_{0}^{(n)}(k, t)+\frac{N_{0}}{N} \frac{1}{N_{1}} \epsilon_{0} p_{0}^{(n)}(k+1, t)
\end{aligned}
$$

where $N_{1} / N$ is the frequency with which we pick a node from the generators group, and $N_{0} / N$ from the destroyers one. The factor $\frac{1}{N_{1}}$ takes into account the fact that we make only one node extraction per timestep and the neighbor degree distribution is the degree distribution of the generator nodes "seen by" a destroyer through one of its randomly chosen links. The variable $\epsilon_{0}$ takes in account the fact that only active destroyers (non-empty nodes) can contribute to the cutting rate, so we have that $\epsilon_{0}=1-\rho_{0}(0, t)$, where $\rho_{0}(0, t)$ is the probability to have picked up an empty destroyer node.

Under the assumption of an uncorrelated network the conditional degree distribution does not depend on the destroyer node with which the generator of degree $k$ is connected. In general, one should know the conditional probability $P(k \mid q)$ that a link emanating from a $q$-degree destroyer is connected to a generator of degree $k$. It is possible to derive the neighbor degree distribution writing the detailed balance condition for a bipartite graph (Kitsak and Krioukov, 2011):

$$
\frac{k}{\langle k\rangle} \rho_{1}(k) P(q \mid k)=\frac{q}{\langle q\rangle} \rho_{0}(q) P(k \mid q)
$$

where $q$ is the destroyer's degree and $\rho_{0}(q)$ is the degree distribution for destroyers. Summing both members of eq.(2) over $q$ the detailed balance condition becomes:

$$
\frac{k}{\langle k\rangle} \rho_{1}(k) \sum_{q} P(q \mid k)=\frac{1}{\langle q\rangle} \sum_{q} q \rho_{0}(q) P(k \mid q)
$$

where, in the case of uncorrelated networks, the conditional probability $P(k \mid q)$ does not depend on $q$ and it can be taken out of the sum. Conditional probabilities satisfy the normalization condition $\sum_{q} P(q \mid k)=1, \forall k$, attracting the attention to the fact that if there are isolated destroyers it is not possible to reach an isolated node from any other generator in the graph. 
Finally, with the hypothesis of no correlation in the network, the conditional probability coincides with the neighbor degree distribution:

$$
P(k \mid q)=p_{0}^{(n)}(k)=\frac{k}{\langle k\rangle} \rho_{1}(k)
$$

At this point it is possible to rewrite the master equation eq.(1) in the general form of a birth-death process as

$$
\begin{aligned}
\rho_{1}(k, t+1) & =\rho_{1}(k, t)+ \\
& +\Gamma^{+}[k-1] \rho_{1}(k-1, t)-\Gamma^{+}[k] \rho_{1}(k, t)+ \\
& -\Gamma^{-}[k, t] \rho_{1}(k, t)+\Gamma^{-}[k+1, t] \rho_{1}(k+1, t)
\end{aligned}
$$

that represents the non-homogeneous add-cut link dynamics in the network. The transition probability can be written as:

$$
\begin{aligned}
\Gamma^{+}[k] & =\frac{N_{1}}{N} \frac{1}{N_{1}} \\
\Gamma^{-}[k, t] & =k \cdot \frac{N_{0}}{N} \frac{1}{N_{1}} \frac{\epsilon_{0}}{\langle k\rangle}
\end{aligned}
$$

where we can use of the bipartite connectivity relation $L=\langle k\rangle N_{1}=\langle q\rangle N_{0}$ is the total number of links in the network.

The master equation for generators is coupled with the master equation for destroyers through the term $\epsilon_{0}$, which takes into account the isolated destroyers in the graph.

The assumption of an uncorrelated bipartite graph is equivalent to the mean field approximation (see Vanni and Barucca (2017) for a more detailed discussion and an alternative derivation of the master equation), which allows one to characterize analytically the behavior of the state variable of each node with respect to the average state variable across the network. In this approximation, the individual interacts with the average individual, even if, under this condition, some network aspects become unobservable (e.g., the degree-correlations).

Moreover, the master equation satisfies the reflecting boundary conditions:

$$
\begin{aligned}
\Gamma^{+}\left[k=N_{0}\right] & =0 \\
\Gamma^{-}[k=0] & =0
\end{aligned}
$$

which take in account the fact that a node cannot have less than zero links, and it has the natural limitation of a fully connected node.

The master equation of the degree distribution reminds one of a nonhomogeneous birth-death process where the rates are time dependent. Following the authors (Bassler et al., 2015), the system reaches a state where the transition rates become homogeneous recovering the detailed balance condition. In any case, we only use the fact that the master equation is restricted under reflecting boundary conditions to a closed system, that is enough to 
guarantee the existence of a stationary distribution regardless of the number of links at the beginning of the process (Gardiner, 2010).

In the steady state, the dynamics of the network is fully described by the bipartite graph between the two groups of agents. So the bipartite model fully describes the evolution of the cutting-adding of links as shown in Fig.1, where a bipartite structure is clear for off-diagonal blocks in the adjacency matrix.

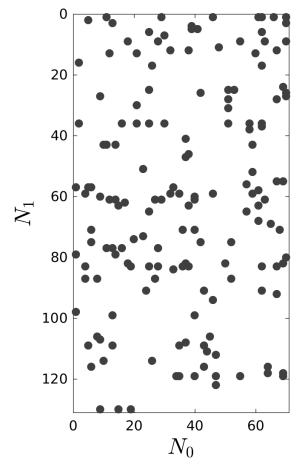

(a) Snapshot at a given time of the adjacency matrix $\mathbf{B}$ of the bipartite target network.

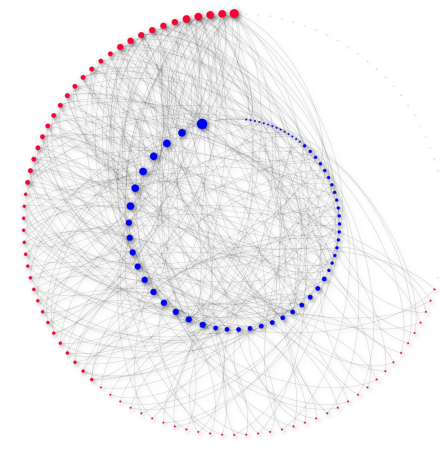

(b) Graphical representation of the bipartite target network at a given time, inner circle nodes are the high target nodes $N_{1}$. Node sizes in the graph are proportional to their degrees.

Fig. 1: The temporal bipartite target network, at a given time step, has a typical adjacency matrix (a) where only the off-diagonal blocks are involved in the dynamics. The same bipartite structure can be visualized in (b) as a core (high target nodes) represented by black circles and the periphery (low target nodes) represented by red circles. Arcs are the links between the two groups made up of $N_{1}=130$ and $N_{0}=70$.

\section{Numerical Solution and results}

In order to have the numerical solution of the degree distribution, it is possible to derive from the master equation (1) the rate equation considering that $\rho_{1}\left(k, t_{n}\right)=\left\langle n_{1}\left(k, t_{n}\right)\right\rangle / N_{1}$ where $\sum_{k=0}^{N_{0}}\left\langle n_{1}\left(k, t_{n}\right)\right\rangle=N_{1}$, under the mean-field approximation. The average $\langle\cdot\rangle$ is the ensemble average over the nodes for given times $t$. It is possible to write the symmetric master equation for the destroyers in terms of "shadow links" which are defined as the total number of possible links minus the actual links. In some sense they are the "imaginary" links which could be created in the network. In this perspective destroyer nodes are generators of shadow links and generator nodes are destroyers of shadow links. In particular, we have that the shadow links are $N_{0} N_{1}-L$ where $L$ is the total number of links in the networks. For a short notation let us indicate $k_{0}:=\langle q\rangle$ and $k_{1}:=\langle k\rangle$. For each destroyer node, the shadow degree is $s=N_{1}-k$ with 
$k=0 \ldots N_{1}$, where we have the fundamental relation for the interdependent average degrees:

$$
s_{0}=N_{1}-k_{1} \frac{N_{1}}{N_{0}}
$$

At this point we can write the coupled equations for the evolution of the network in terms of the two interacting populations as:

$$
\begin{aligned}
\left\langle n_{1}\left(k, t_{n}+1\right)\right\rangle & =\left\langle n_{1}\left(k, t_{n}\right)\right\rangle+ & \left\langle n_{0}\left(s, t_{n}+1\right)\right\rangle & =\left\langle n_{0}\left(s, t_{n}\right)\right\rangle+ \\
& +\frac{N_{1}}{N} \frac{\left\langle n_{1}\left(k-1, t_{n}\right)\right\rangle}{N_{1}}+ & & +\frac{N_{0}}{N} \frac{\left\langle n_{0}\left(s-1, t_{n}\right)\right\rangle}{N_{0}}+ \\
& -\frac{N_{1}}{N} \frac{\left\langle n_{1}\left(k, t_{n}\right)\right\rangle}{N_{1}}+ & & -\frac{N_{0}}{N} \frac{\left\langle n_{0}\left(s, t_{n}\right)\right\rangle}{N_{0}}+ \\
& -\frac{N_{0}}{N} \frac{k\left\langle n_{1}\left(k, t_{n}\right)\right\rangle}{N_{0}} \frac{\epsilon_{0}}{k_{0}}+ & & -\frac{N_{1}}{N} \frac{s\left\langle n_{1}\left(s, t_{n}\right)\right\rangle}{N_{1}} \frac{\epsilon_{1}}{s_{1}}+ \\
& +\frac{N_{0}}{N} \frac{(k+1)\left\langle n_{1}\left(k+1, t_{n}\right)\right\rangle}{N_{0}} \frac{\epsilon_{0}}{k_{0}} & & +\frac{N_{1}}{N} \frac{(s+1)\left\langle n_{0}\left(s+1, t_{n}\right)\right\rangle}{N_{1}} \frac{\epsilon_{1}}{s_{1}}
\end{aligned}
$$

In order to get the full description of the model, the number of empty destroyer and generator nodes can interchangeably be obtained from the master equations as:

$$
\epsilon_{0}=\frac{N_{0}-\left\langle n_{0}\left(k=0, t_{n}\right)\right\rangle}{N_{0}} \quad \mid \epsilon_{1}=\frac{N_{1}-\left\langle n_{1}\left(s=0, t_{n}\right)\right\rangle}{N_{1}}
$$

where we can observe that number of fully connected generator nodes is the same as the number of isolated generators in terms of shadow links, i.e., $\left\langle n_{1}\left(k=N_{0}\right)\right\rangle=\left\langle n_{1}(s=0)\right\rangle$.

The process description is valid for $k=1 \ldots N_{0}-1$, and the boundary conditions at $k=0$ and $k=N_{0}$ are:

$$
\begin{aligned}
& \left\langle n_{1}\left(k=0, t_{n}+1\right)\right\rangle=\left\langle n_{1}\left(0, t_{n}\right)\right\rangle+ \\
& -\frac{\left\langle n_{1}\left(0, t_{n}\right)\right\rangle}{N}+ \\
& \left\langle n_{0}\left(s=0, t_{n}+1\right)\right\rangle=\left\langle n_{0}\left(0, t_{n}\right)\right\rangle+ \\
& +\frac{\left\langle n_{1}\left(1, t_{n}\right)\right\rangle}{N k_{0}} \epsilon_{0} \\
& \left\langle n_{1}\left(k=N_{0}, t_{n}+1\right)\right\rangle=\left\langle n_{1}\left(N_{0}, t_{n}\right)\right\rangle+ \\
& -\frac{\left\langle n_{0}\left(0, t_{n}\right)\right\rangle}{N}+ \\
& +\frac{\left\langle n_{0}\left(1, t_{n}\right)\right\rangle}{N s_{1}} \tilde{\epsilon} \\
& \left\langle n_{0}\left(s=N_{1}, t_{n}+1\right)\right\rangle=\left\langle n_{0}\left(N_{1}, t_{n}\right)\right\rangle+ \\
& +\frac{\left\langle n_{1}\left(N_{0}-1, t_{n}\right)\right\rangle}{N}+ \\
& +\frac{\left\langle n_{0}\left(N_{1}-1, t_{n}\right)\right\rangle}{N}+ \\
& -\frac{N_{0}\left\langle n_{1}\left(N_{0}, t_{n}\right)\right\rangle}{N k_{0}} \epsilon_{0} \\
& -\frac{N_{1}\left\langle n_{0}\left(N_{1}, t_{n}\right)\right\rangle}{N s_{1}} \tilde{\epsilon}
\end{aligned}
$$

In our simulations we took the initial network to be empty, i.e., $n_{1}(k, t=$ $0)=N_{1} \delta_{0, k}$.

So the only parameter that has an impact on the statistical structure of the network is the ratio between the two populations.

As results of our mathematical interpretation of the model we compare the degree distributions obtained by the Monte Carlo simulation of the model with 
the distributions obtained as numerical results of the coupled master equations. As expressed in appendix A, the distributions obtained by the master equation approach are bounded poisson distributions.

According to the values of $N_{0}$ and $N_{1}$ the network behaves differently, so we define as control parameter of the system the quantity

$$
h=\frac{N_{1}-N_{0}}{N_{1}+N_{0}}
$$

that is the proportion variable between the two groups.

In Fig. 2 the degree distribution of the network is plotted for several values of $N_{1}$ and $N_{0}$. It is evident that the model prediction is exact when considering the first moment of the distribution (the expected value of the degree). This is a consequence of the first-order approximation made in the master equation representation using a mean field approach. As regards the entire degree distribution, it is exact for all the values of $h$ but when we are at the values for which $N_{1}=N_{0}$ : here the degree distribution departs from the poisson distribution and is closer to a fatter distribution whose mean is equal to the estimated average degree $\mu=\langle k\rangle_{1}$ but the variance is actually of the order of the support of the distribution $\sigma^{2} \sim N^{2}$.

All these results are in line with the solutions obtained in (Bassler et al., 2015) via numerical convergence of a fixed-point procedure; the resultant degree distributions have been compared numerically with the master equation solution. We replicated both approaches and compared them with respect to the true model distributions: We obtained the same Kullback-Leibler divergence up to a factor of $10^{-6}$ as analyzed in appendix A.

Using the interacting master equations we can also obtain and plot the temporal evolution of the degree distributions and consequently the average time needed to reach the associated stationary distributions, as well illustrated by Fig.3.

The same type of results are obtained in the evaluation of the number of empty low-target nodes and the full high-target ones, see Fig.4.

Once we numerically obtain the value of the average degree and the number of empty nodes, we can recover the shape of the degree distribution as a bounded poissonian distribution (see appendix A):

$$
\rho_{1}(k)=\frac{1}{e^{\chi \frac{\Gamma\left(N_{0}+1, \chi\right)}{\Gamma(N 0+1)}-1}} \frac{\chi^{k}}{k !} \quad, k=0 \ldots N_{0}
$$

where $\chi=\frac{N_{1}}{N_{0}-n_{00}} k_{1}$, and $n_{00}=\left\langle n_{0}\left(0, t_{n}\right)\right\rangle$ is the average number of empty destroyer nodes.

Other than the node degree, we can also characterize the network formation in terms of the effective degree:

$$
\xi=\frac{k}{N_{0}}, \quad \in[0,1]
$$




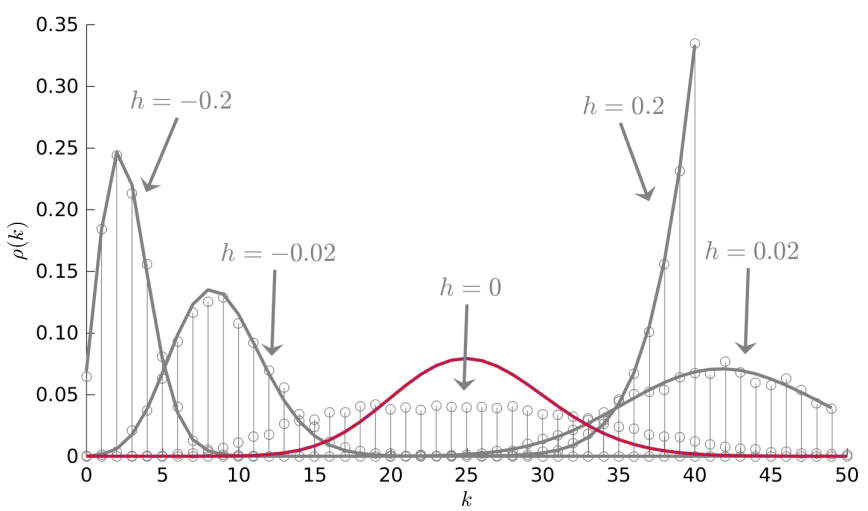

Fig. 2: In a network of $N=100$ nodes, using ensemble averages of 1000 samples. Where $h=\left(N_{1}-N_{0}\right) / N$ is the population control parameter. The stem plot represents the degree distribution from model simulations of the target network, the continuous gray lines are degree distributions obtained directly from the coupled master equations. The two distributions always coincide in shape and are centered exactly at the average degree values. An exception happens for populations such that $N_{1}=N_{0}(h=0)$, here the approximated distribution (dashed line) is less fat then the real distribution. This is a direct consequence of the mean-field approximation made in the master equation representation of the model.

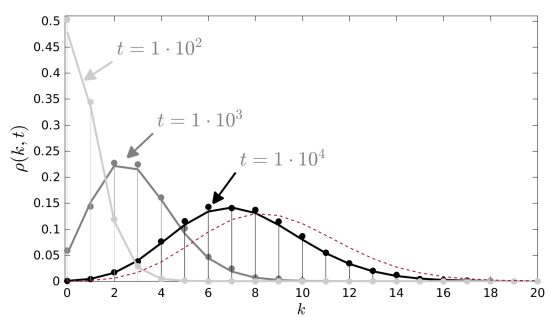

(a) Stem plot distributions are from the Monte Carlo simulations of the model, the continuous lines are the distributions from the master equation. Different time snapshots show the agreement between the two approaches. The dashed curve represents the stationary distribution.

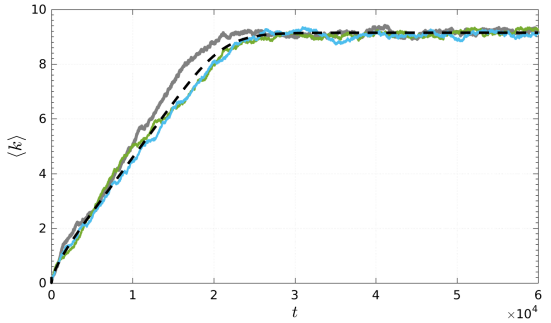

(b) The three lines are three different realizations of the model, the black dashed line represents the time-evolution of the average degree in numerical solutions of the master equation. The dotted curve represents the stationary degree distribution.

Fig. 3: Time evolution of the degree distribution (a) and the average degree (b) of the bipartite network simulations and their mathematical counterparts, the coupled master equations, for the case of $N_{1}=51, N_{0}=49$ where $h=-0.02$. Notice how good the approximations are maintaining both the distribution shape and detecting the right average convergence time to the equilibrium distribution.

which is a normalized degree measure; it measures the number of links created by a high-target node as a fraction of the total number of possible links it can make (i.e., $N_{0}$ ). The average over all the high-target nodes gives the network degree efficiency $\langle\xi\rangle$ : values close to 0 indicate that the high-target nodes create very few links with respect to the possible links they can make. On the 
other hand, values of the efficiency close to 1 indicate a high capability for each high-target node to generate links with the other group's nodes. The passage between these two extreme situations exhibits a phase transition, where we have an abrupt change in efficiency when $N_{1}=N_{0}$.

We plot the phase transition in Fig.5. Exploring the average value of the mean field variable $\xi$ for all the possible values of the populations of the two groups, we have a phase transition, where the critical point is the case $N_{1}=$ $N_{0}$, in which we have a big jump from a minimally-efficient network to a maximally-efficient network. The figure shows that the numerical solution of the coupled master equations approach gives the exact behavior of the phase transition, which accounts only for the first moment of the distribution.

Notice that the phase transition is a property of the network efficiency and not of the average degree by itself. In any case, they share the same emergent properties mostly due to a second-order effect related to the variance of the average degree and the node correlation in the network.

\section{Criticality: Correlations and Burstiness}

Despite the presence of a phase transition, the emergent properties of the target network reside in higher moments of the degree distribution, those which are not satisfactorily addressed when the network is at criticality. The first important emergent property is the variance of the degree distributions, which exhibits an abrupt large value at criticality $(h=0)$. We want to give some heuristic explanation of this phenomenon. 


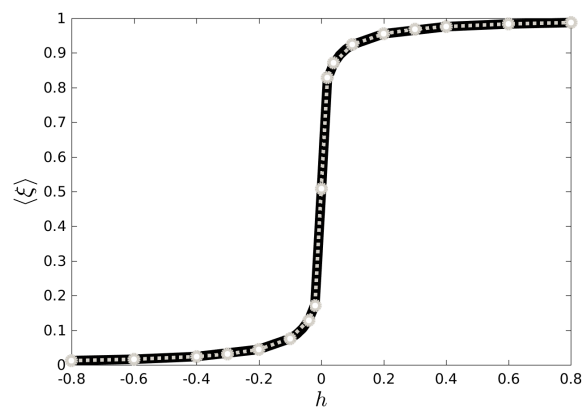

Fig. 5: Phase transition of the mean value of the mean-field variable $\langle\xi\rangle$ (the network efficiency) over the possible values of $N_{1}$ and $N_{0}$ in a bipartite network with $N=100$ total nodes. The bold black line is the curve corresponding to the model simulation, the dashed white line is the curve found using the coupled master equations. The plot suggests the idea that, in a target-network, a node being part of a group endogenously increases (or decreases) its ability to follow its preference.

\subsection{Degree Correlations}

The stochastic process associated to the the network dynamics can also be analyzed through the time series of the average degree as in Fig.6, which shows a given mean value and a standard deviation.

Given the ergodicity of the process we can freely interchange the mean value over time and the average over different trajectories. Finally, we can build the two types of distributions: the degree distribution and the averagedegree distribution built along its trajectory. We plot these probabilities in Fig.7.

The two distributions, and their moments as well, are related by convolution of the distributions of each node, since $\langle k\rangle=\frac{1}{N_{1}} \sum_{i=1}^{N_{1}} k_{i}$, defining a sort of a sampling distribution for the mean. The expected value is

$$
E[\bar{k}]=\langle k\rangle
$$

where $\bar{k}$ is the mean degree calculated over a single trajectory, and the variance of the mean degree is:

$$
\begin{aligned}
\operatorname{Var}(\bar{k}) & =\frac{1}{N_{1}^{2}} \sum_{i=1}^{N_{1}} \operatorname{Var}\left(k_{i}\right)+\frac{1}{N_{1}^{2}} \sum_{i=1}^{N_{1}} \sum_{j \neq i}^{N_{1}} \operatorname{Cov}\left(k_{i}, k_{j}\right) \\
& =\frac{\sigma^{2}}{N_{1}}+\frac{\sigma^{2}}{N_{1}^{2}} \sum_{i=1}^{N_{1}} \sum_{j \neq i}^{N_{1}} \rho_{k_{i}, k_{j}} \\
& =\frac{\sigma^{2}}{N_{1}}+\rho \frac{N_{1}-1}{N_{1}} \sigma^{2}
\end{aligned}
$$

where $\rho_{k_{i}, k_{j}}$ is the cross-correlation coefficient between nodes $i$ and $j$ under the hypothesis the variables have equal $\sigma^{2}$, which is the variance of the degree of 


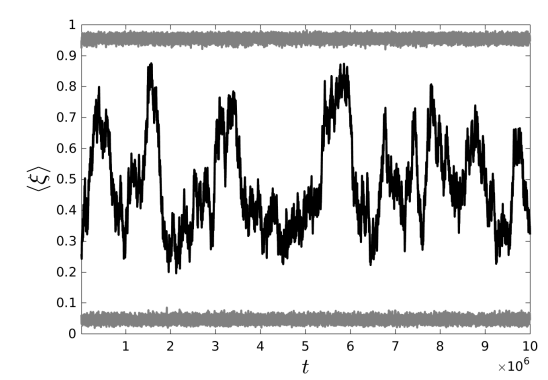

(a) Temporal behavior of the average degree or equivalently of the degree efficiency $\langle\xi\rangle=\langle k\rangle_{1} / N_{0}$ where $N=100$ : the black central line represents the meanfield parameter $\xi$ in the equal proportion case of $N_{1}=N_{0}=50$ in which we have a "quasi"-free diffusion process which explores all the the every values of the nodes degree. The top gray line is the case $N_{1}>N_{0}=45$, and the bottom gray line is for the case $N_{1}<N_{0}=55$. In these two cases the brownian motion is attenuated by the reflecting boundary condition, resulting in much smaller fluctuations.

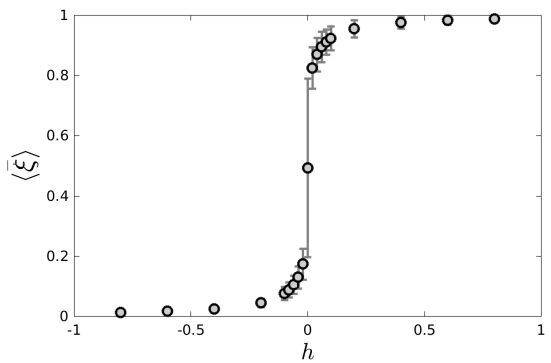

(b) The phase transition of the degree efficiency, this time the error bars represent twice the standard deviation of the relative time series. The huge amplitude of the fluctuations at criticality is evident, thus defining the onset of an emergent property of the network when the systems is at the critical point $N_{1}=N_{0}$.

Fig. 6: The average degree and the relative efficiency variable can also be described in terms of their time series as a function of simulation time as in (a). This is equivalent to evaluating several times the average degree for different trajectories at a given time. The resulting variable is $\overline{\langle k\rangle}$ that is the mean degree, in (b) we also plotted the error bars which relate to the standard deviation of the average degree as a function of time.

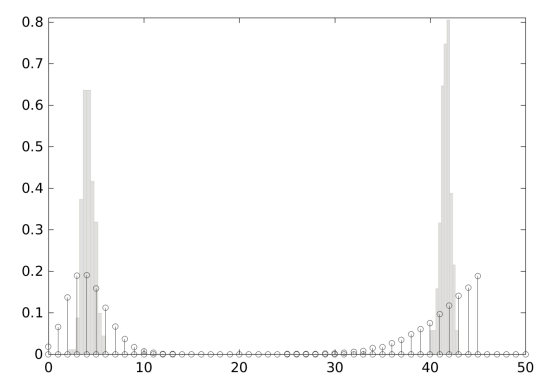

(a) The two distributions on the left are for $N_{1}=45, N_{0}=55$ in the sub-critical case, the two right distributions are for $N_{1}=55, N_{0}=45$ in the super-critical regime.

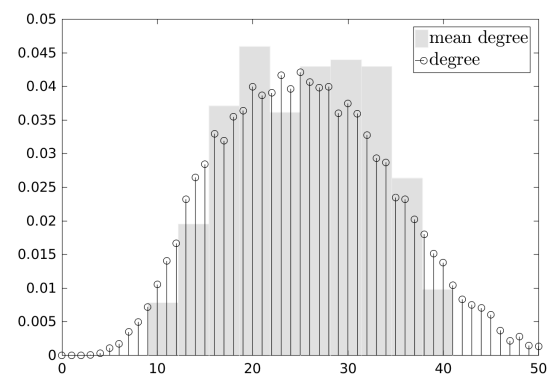

(b) The special case of criticality $\left(N_{1}=\right.$ $N_{0}$ ), where the two distributions are both fat with the same mean and different variances.

Fig. 7: Comparison between two distributions: stem black lines indicate the ordinary degree distribution $\rho(k)$ and the gray bars indicate the sampling distribution of the average degree. (a) is the case in which $N_{1} \neq N_{0}$ and (b) the case $N_{1}=N_{0}$ at criticality. 
each node (generator), $\operatorname{Var}(\bar{k})$ is the variance of the average degree distribution (the sampling distribution), and $\rho$ is the average value of the $\left(\begin{array}{c}N_{1} \\ 2\end{array}\right)$ correlation coefficients $\rho_{k_{i}, k_{j}}$. If the nodes were independent, the average correlation factor $\rho$ would be zero. Actually, from the empirical studies of the model we can estimate the value of the correlation factor $\rho$, by comparing the variances of the two distributions evaluated via simulations

$$
\rho=\frac{\operatorname{Var}(\bar{k})}{\sigma^{2}} \frac{N_{1}}{N_{1}-1}-\frac{1}{N_{1}-1}
$$

which for large networks approaches the average ratio between the variance of the sample mean distribution over the variance of the degree distribution. What is obtained is well expressed in Fig.8, where strong correlations arise among the nodes at criticality, i.e., $N_{1}=N_{0}$. This is evidence that it is not possible to ignore the presence of node degree correlations in the mathematical description of the model. For example, the model prescription prevents a node from going beyond its capacity, so that if a low-target node is already full, a high-target node is forced to exclude it from its neighbour candidates. On the

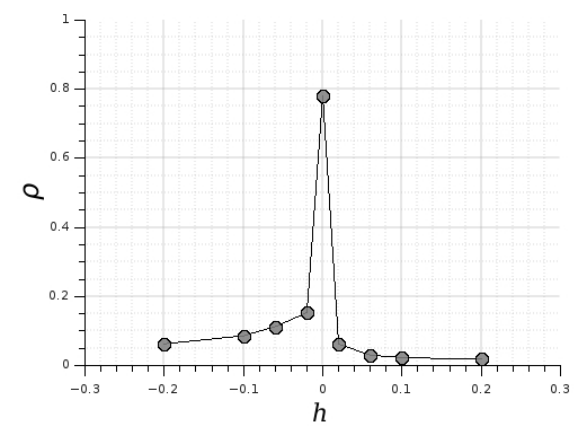

Fig. 8: Average correlation estimation obtained comparing the sampling distributions of the average degree with the ordinary degree distributions. It is evident that at criticality $(h=0)$ the correlation is much higher then in the off-critical cases $(h \neq 0)$. This shows how neglecting the correlation among the nodes makes the master equation approach fail at criticality.

contrary, the master equation which describes the network dynamics has been addressed using a first-order mean field approximation. In evaluating the cutting rates we only consider first-order effects ignoring any possible correlation among the nodes in selecting the nodes to connect with.

In the case of the critical condition $N_{1}=N_{0}$, the mean-field approximation fails and the conjecture of a degree-uncorrelated network cannot be applied. In particular in the critical case, the conditional degree probability $P(k \mid q)$ it is not independent of the destroyer node picked. From eq.(2), in the presence of degree correlations we expect $P(k \mid q) \neq p_{0}^{(n)}(k)$, so the degree distribution 
of generators "seen by" a destroyer is not the neighbor degree distribution $p_{0}^{(n)}(k)$ as in the uncorrelated case.

To better investigate the nature of such macroscopic correlation effects, we make use of the traditional definition of assortativity intended to quantify the degrees correlations. We make use of the average nearest-neighbor degree (ANND), which is the average degree of the neighbors of all $k$-degree nodes (Pastor-Satorras et al., 2001). When dealing with a bipartite network, it is possible to measure degree correlations both with respect to generator nodes and with respect to destroyer nodes, defining (Saracco et al., 2015) the average nearest neighbors' degree (ANND):

$$
\begin{aligned}
k_{n n, i}(\mathbf{B}) & =\frac{\sum_{j=1}^{N_{0}} B_{i j} q_{j}}{\sum_{j=1}^{N_{0}} B_{i j}} \\
q_{n n, j}(\mathbf{B}) & =\frac{\sum_{i=1}^{N_{1}} B_{i j} k_{i}}{\sum_{i=1}^{N_{1}} B_{i j}}
\end{aligned}
$$

where $\mathbf{B}$ is the adjacency matrix of the bipartite graph, $k_{i}$ is the degree of the $i$-th generator node and $q_{j}$ is the degree of the $j$-th destroyer node. The assortativity of the bipartite network is quantified by plotting the ANNDs values versus the degree sequences $\left\{k_{i}\right\}_{i=1}^{N_{1}}$ and $\left\{q_{j}\right\}_{j=1}^{N_{0}}$ to depict the overall assortativity trend for a network

The average nearest neighbors' degree functions, in our bipartite network, are connected to the conditional probability with $q_{n n}(k)=\sum_{q} q P(q \mid k)$ and $k_{n n}(q)=\sum_{k} k P(k \mid q)$. In the uncorrelated case we have the constant values (Kitsak and Krioukov, 2011):

$$
\begin{aligned}
& q_{n n}(k)=\frac{\left\langle k^{2}\right\rangle}{\langle k\rangle} \\
& k_{n n}(q)=\frac{\left\langle q^{2}\right\rangle}{\langle q\rangle}
\end{aligned}
$$

so that the average nearest neighbors' degree is equal to the average degree of the node to which a link leads. In the critical case we have $\langle q\rangle=\langle k\rangle$, so the two equations coincide. This is the case of neutral assortativity where no correlations are present in the network, since the ANND is independent of the degree $k$. While in the off-critical case eqs.(26) (27) are always satisfied, in the critical condition the study of the ANND deserves a more delicate approach. First of all, we run many simulations on the model to study the correlation structure according to the dependence of the ANND on the degree. In particular, we study the average of the ANND over nodes with the same degree $\bar{k}_{n n}=\sum_{i=1}^{N_{1}} k_{n n} / N_{1}$. As shown in Fig.9, we study the mean-ANND $\bar{k}_{n n}$ over time and the ANND function $k_{n n, i}(k)$ at particular time snapshots. We deduce that at each time step the network is uncorrelated, the average value of ANND fluctuates over time following perfectly the average degree $\langle k\rangle$. In some sense, according to a pointwise evaluation of the correlations, 
the network is uncorrelated but the conditional probability (and the neighbor probability $\left.p_{0}^{(n)}(k)\right)$ is dependent on time.

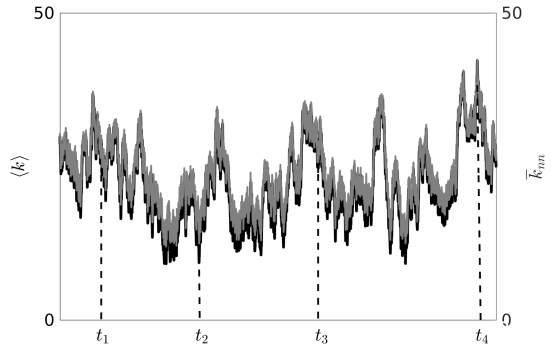

(a) Comparison between the fluctuationg behavior of the average degree (black line, left vertical axes) and the mean-ANND (grey line, right vertical axes) over time. The mean-ANND (average degree of the nearest neighbor of a node) time series has a delay of the unit numerical step in comparison with the average degree series. We notice that the mean value of the ANND is equal to the value of ANND in the uncorrelated case.

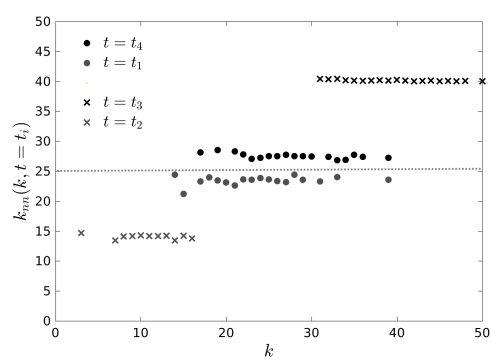

(b) ANND function for four different times $t_{i}$. At each timestep the network is uncorrelated, while the ANND changes value according to time. The dashed horizontal line represents the case of an uncorrelated network.

Fig. 9: Neighbor connectivity for the system at the critical point $N_{1}=N_{0}=50$. In (a) we show a two axis plot where the fluctuations of the average degree are compared to the meanANND. In (b) we study the nearest neighbor average degree function at different times. We notice that for $k=40$ we have 3 different values of the ANND.

As for temporal assortativity, we use a way to represent the aggregate average nearest neighbor degree function (a-ANND). First of all, for each time snapshot $t$ we define the event $E(k, t)$ the case where the network has node with degree $k$ for which we can compute the ANND. At this point we define an aggregate measure of assortativity: the mean value of the $\operatorname{ANND}(\mathrm{k})$ over the times we have observed the events $E(k, t) \forall t=1 \ldots T$ :

$$
f_{k}=\frac{1}{N_{k}} \sum_{t=1}^{T} k_{n n}(k, t)=:\left\langle k_{n n}(k)\right\rangle_{T}
$$

where $N_{k}=\sum_{t=1}^{T} E(k, t)$ is the number of times we have observed at least one node with degree $k$ in a total number of $T$ scatter plots ${ }^{1}$. For example, in figure Fig.9b for $k=40$, we have 3 values of $k_{n n}(k=40)$, whose average is $\left(k_{n n}\left(40, t_{3}\right)+k_{n n}\left(40, t_{4}\right)+k_{n n}\left(40, t_{1}\right)\right) / 3$, that is about 30.67 in that case.

\footnotetext{
1 Note the the normalization factor of the mean is not $T$, since we do not count the case in which there are no nodes with a given ANND for a time snapshot $t$. In the case in which in each timestep we always find an ANND for the associated degree $k$ then we have $N_{k}=T$. However, in general, this is not the case.
} 
The scatter plot $\left\{f_{k}, k\right\}_{T}$ gives the aggregate ANND function over an observation time-window of length $T$, see Fig.10. If the process is ergodic after a sufficiently large observational time, the aggregate ANND is independent of $T$.

In conclusion, we showed that in the case $N_{1} \neq N_{0}$ the network is uncorrelated since it has neutral assortativity for every time step. At the critical point of the connectivity phase transition $N_{1}=N_{0}$ the uncorrelation assumption does not work since the degree correlations, despite their pointwise neutral assortativity, change over time with an aggregate effect on the macroscopic description of the model.

The result of such overall temporal effects is not captured by a static mean field approximation, but it could be incorporated by taking into account the aggregate ANND function over time.

To describe the system at criticality, in principle, one should know the time-dependent conditional probability $P_{t}(k \mid q)$ and so fully characterize the correct degree-degree correlations. In an aggregate picture, we move from a time dependent description to a degree-dependent one.

From a global level, we see that the system appears to generate an aggregate correlation effect with non-neutral assortativity as is clearly evident from Fig.10, where the aggregate ANND is a linear function $\left\langle k_{n n}(k)\right\rangle_{T}=\beta k$ which underlines the existence of a positive assortative bipartite network: this means that only nodes with the same degree are connected with each other. We have seen that when $N \rightarrow \infty$, the proportionality constant $\beta \rightarrow 1$, yielding a perfect positive assortativity in the thermodynamic limit.

The fact that the degree correlations are so intense is equivalent to approximate the neighbor distribution by $\tilde{p}_{0}^{(n)}(k) \sim \rho_{1}(k)$, meaning that the probability of picking a destroyer which cuts a link with a generator of degree $k$ is the same as randomly picking a generator of degree $k$ and randomly cutting one of its links. In the limiting case of a very large network $(N \rightarrow \infty)$, the assortative mixing is perfect, so the neighbor probability is exactly the same as the degree distribution. Using this approximation in the master equation, we can write:

$$
\rho_{1}^{s t}(k)=\frac{2}{N+2}
$$

which reminds one of the stationary distribution of a random walk with reflecting barriers. In a finite network we have a strong boundary effect where the strong correlations vanish (see Appendix A.2 for a more detailed discussion and numerical approximations).

\subsection{Bursty events dynamics}

In the following section we investigate on the bursty nature of the network dynamics showing how the fluctuations and correlations induce a temporal property related to the distribution of inter-event times between two consecutive passages from a sparse graph to a dense one (and vice-versa). 


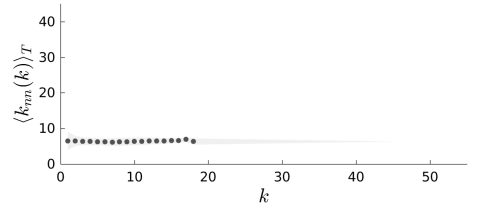

(a) $N_{1}=55$. No aggregate assortativity, the bipartite graph is correlation neutral.

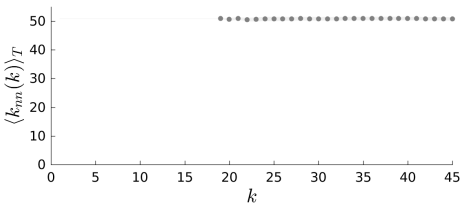

(b) $N_{1}=45$. No aggregate assortativity, the bipartite graph is correlation neutral.

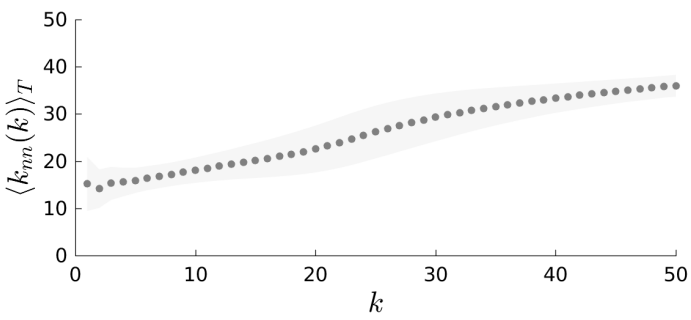

(c) $N_{1}=N_{0}$, criticality. Clear overall degree correlation, since the aggregate ANND has increasing assortative mixing. The linear dependence is given by $\left\langle k_{n n}(k)\right\rangle_{T}=\beta k$ with $\beta=0.5$.

Fig. 10: Aggregate assortativity over time. This figure expresses how in our macroscopic description of the network (master equation) the system appears strongly correlated, since the dependence of the ANND with respect to the degree $k$ is not constant. The sampling has been addressed for a network with $N_{1}=N_{0}=50$ over a sampling time of $T=3 \cdot 10^{7}$. The shaded area represents an error around the mean of two standard deviation of the average ANND for each $k$. In the off-criticality case the error is negligible.

We define the inter-event time as the time between two consecutive events on the connectivity property of the network. The property under discussion is the density of links defined as connectance ${ }^{2}$ :

$$
\ell=2 \frac{\langle k\rangle_{1} N_{1}}{N_{0} N_{1}}-1 \quad \text { with } \ell \in[-1,1]
$$

which is the actual average number of links over the possible number of links. The connectance has values close to -1 when the network is sparse and it has values close to 1 when the network is dense.

As illustrated in Fig.11, the definition of an event is related to the condition when the network passes from a sparse (dense) connectivity to a dense (sparse) one. The $i$-th inter-event time, denoted by $\tau_{i} 1 \leq i \leq T-1$ where $T$ is the length of the time series, is defined by:

$$
\tau_{i}=t_{i+1}-t_{i}
$$

2 The connectance is defined as the link density of the network, which is the fraction of the number of actual links over the number of potential links between pairs of nodes. By the term connectivity, we indicate a generic property which gives a measure of the number of links in the network. 


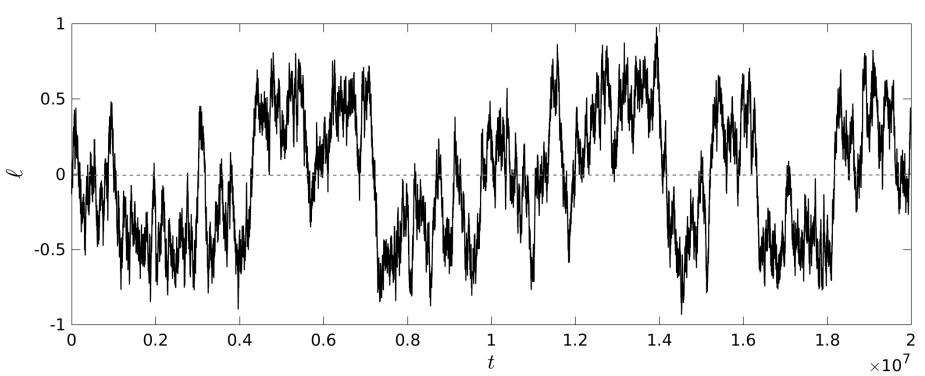

(a) Fluctuations of the network connectance at criticality $(h=0)$. It is possible to notice how the system goes through periods of high connectivity (for $\ell>0$ ) to periods of low connectivity (for $\ell<0$ ).

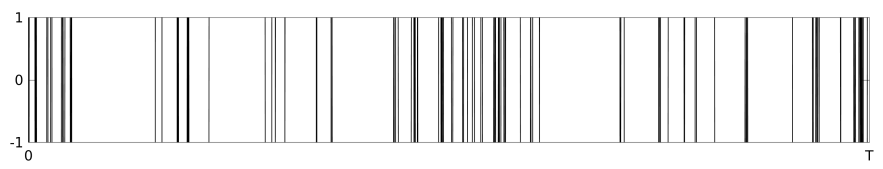

(b) We display the event sequence among the switching states of sparse and dense network.

Fig. 11: The construction of the event sequence from the fluctuations of the network connectance as in (a). We detect an event when the fluctuations goes from a period of sparse condition to a condition to dense one and vice-versa, i.e. the periods among two consecutive zero-crossings of the time-series are the inter-event times $\tau_{i}$.

The bursty event sequence $\tau_{i}$ shown in Fig.11b can be treated computing the burstiness as used in the network community (Goh and Barabási, 2008):

$$
B=\frac{\frac{\sigma(\tau)}{\langle\tau\rangle}-1}{\frac{\sigma(\tau)}{\langle\tau\rangle}+1}
$$

where the $\sigma=\sqrt{\frac{1}{T-1} \sum_{i=1}^{T-1}\left(\tau_{i}-\langle\tau\rangle\right)}$ is the standard deviation of $\left\{\tau_{i}\right\}$ and the average inter-event time is $\langle\tau\rangle=\frac{1}{T-1} \sum_{i=1}^{T-1} \tau_{i}$.

An extremely bursty event sequence yields value of the burstiness $B \rightarrow 1$, on the contrary in the case of Poisson processes the burstiness coefficient is zero; in the case of periodic event sequence $B=-1$.

Montecarlo simulations confirm the bursty nature of the network at criticality where, for example in the case of $N=100$, we obtain a burstiness coefficient $B=0.89$, and it tends to 1 when $N \rightarrow \infty$.

Another particularly interesting property of such network model is the presence of heavy-tailed behaviors in the inter-event time distributions. The probability density function $\psi(\tau)$ has been evaluated in terms of its complementary cumulative distribution $\Psi(\tau)=\int_{t}^{\infty} d \tau \psi(\tau)$. As shown in Fig.12 where the power-law distribution of inter-event times is:

$$
\psi(\tau) \propto \tau^{-\alpha}
$$


with $\alpha=1.5$ showing a clear distribution described by power-law decaying behavior.

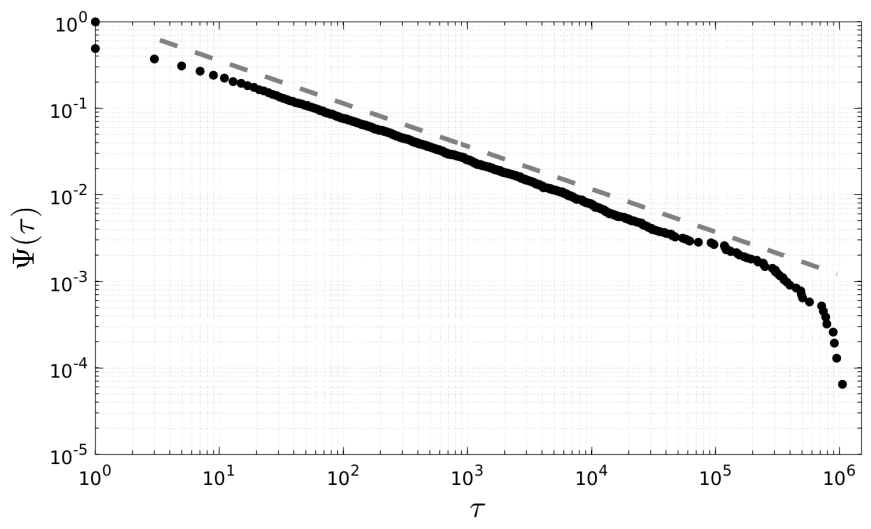

Fig. 12: Complementary cumulative distribution $\Psi(\tau)$ of the inter-event times of the network at criticality. It is clear a power-law distribution with a coefficient of $\alpha=1.5$ in eq.(33).

\section{A more general model: intermittent system and connection rate}

In the present section we show a more general model which takes in account the role of passive nodes which in principle can have an important role in the network dynamics.

As an extension of the previous model, we provide a more plausible agentdriven network based on the XIE model where the unilateral initiatives are smoothed by the possibility that the unit does not manage to act according to its attitude of preferred degree.

Specifically, let us take into account the chance that the action of the agent to generate (or destroy) a link is not accomplished. So that, for various reasons, the connection (or de-connection) attempt fails, for example because of the opposition of the counterpart agent. So whenever a unit is drawn, it tries to act according its preference, adding or cutting a link, but here we consider the chance that this attempt fails with a rate $\lambda$ (target-failure probability). It is possible to generalize the CMED approach just rewriting the master equation as in eq.(1) but taking into account two modes of the degree motion, the add-cut process phase and the resting phase that justifies the presence of "idle" states due to the missed action of the node tendency. Consequently, we consider the population density at rest $\rho_{1}^{(i)}(k, t)$ in addition to the population 
density in motion $\rho_{1}(k, t)$, and we can rewrite the CMED equation as:

$$
\left\{\begin{aligned}
\rho_{1}(k, t+1)-\rho_{1}(k, t)= & \Gamma^{+}[k-1] \rho_{1}(k-1, t)-\Gamma^{+}[k] \rho_{1}(k, t)+ \\
& -\Gamma^{-}[k, t] \rho_{1}(k, t)+\Gamma^{-}[k+1, t] \rho_{1}(k+1, t)+ \\
& -\lambda \rho_{1}(k, t)+\lambda \rho_{1}^{(i)}(k, t) \\
\rho_{1}^{(i)}(k, t+1)-\rho_{1}^{(i)}(k, t) & =\lambda \rho_{1}(k, t)-\lambda \rho_{1}^{(i)}(k, t)
\end{aligned}\right.
$$

with the usual transition rates eqs.(6),(7) and boundary conditions as in eqs (8),(9). This new master equation describes the intermittent add-cut process where idle states introduce pauses in the process due to the probability of each node to fail in their intention. Here we considered that the failure rates are the same for all the units in the network. The existence of a stationary solution is guaranteed by the closed domain and the intermittent phases do not contribute to the steady state degree distribution ${ }^{3}$. Consequently, we have the same truncated poisson stationary distribution as in the classical case of XIE. In principle we can also consider different failure rates for the two groups $\lambda_{1}$ and $\lambda_{0}$, then the degree distributions differ from the standard case of XIE. Finally, the generalized network model shows the same degree distribution of the original CMED but the process is slowed down by the connection failures as shown in our numerical simulations of Fig.13; in this case, the average degree trajectories are now time series where "pauses" arise in correspondence to connection failures. This in practice introduces a delay in reaching the equilibrium state as well as a slower speed in the linking mechanism.

The introduction of a failure rate has the purpose to stress the importance of the novelty of the present network mechanism made up of relations between a proposer and a responder. Changing the values of $\lambda$ for different nodes it is possible to obtain a more complex network structure with the purpose to make the model more and more realistic.

\section{Applications in economics and finance}

The nature of the network discussed in this paper displays particular intrinsic features which make it potentially suitable for many applications in economics. The temporal network structure could, indeed, represent an appropriate framework for systems where fluctuations induce a bursty dynamics and emergent degree correlations.

The model of network formation presented here, or similar generalizations, may contribute to our understanding of sudden freezes in financial markets,

\footnotetext{
3 the stationary degree distribution can also be recovered just using the ordinary CMED approach as in eq.(1) and using an event-based representation of temporal networks (Masuda and Lambiotte, 2016). We can here modify the transition rates defining: $\tilde{\Gamma}^{+}[k-1]=\Gamma^{+}[k-$ $1](1-\lambda)$ and $\tilde{\Gamma}^{-}[k, t]=\Gamma^{-}[k, t](1-\lambda)$. The problem with this approach is that we ignore the idle states and that the process has pauses in its trajectories. But, in the end, it reaches, with different transient times, the same degree distribution as using the intermittent CMED.
} 


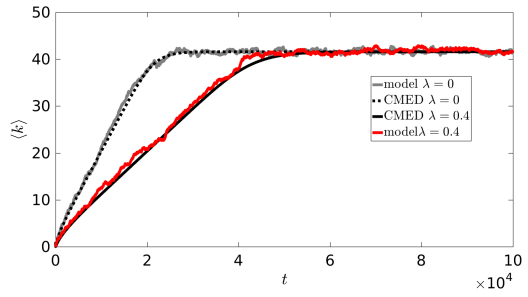

(a) Transition to equilibrium obtained using the model and the CMED in two cases: standard model with no failure rate, and the intermittent case where the failure target probability is $\lambda=0.4$. The CMED approach approximates well both cases, and the introduction of a $\lambda$ makes the system slower to reach the stationary solution for the average degree.

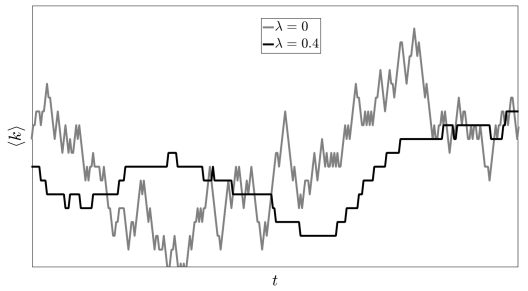

(b) An example of the trajectories for large times, where we notice the presence of pauses in the case where a failure rate is present. This slows down the system but at large times it gives the same degree distribution as the standard model $(\lambda=0)$.

Fig. 13: Trajectories of the average degree for the agent-driven network with target failures and the relative intermittent CMED description. In (a) we notice that the two models and their numerical solutions coincide, reflecting that the introduction of failure probability $\lambda$ slows down the transient state and the reaching of the equilibrium state. The speed factor $\lambda$ does not affect the final degree distribution but, as shown in (b), introduces pauses in the trajectories of the average degree over time thus making the system slower

when agents collectively stop trading a specific instrument between each other after a small change in market conditions. In particular, the mixed transition at criticality may provide an explanation for the abrupt changes in financial behavior during market crisis. In fact, during financial crisis, institutions may suddenly change their strategy and trigger a non-linear response of the whole market - leading to fire-sales, liquidity, inability to acquire funds - through mechanisms similar to the one outlined above. This phenomenon will be the subject of further works that are beyond the scope of the present one. A possible research line has been addressed by Vanni et al. (2018) where an agent based model has been built upon the framework of the network model presented here. In particular, the system represents a financial interbank market in a marked-to-market structure where agents are driven by leverage-risk coupled actions, and where the interactions among the agents are modulated by a temporal complex network with a non-simultaneous link formation rationale. In the market, banks behavior on leveraging actions depends on their perceived risk in terms of a Value at Risk measure as risk management to control their exposure to the stock market. In such an agent based model the population parameter $h$ in eq.(16) of the network is not constant, instead it fluctuates according to the leverage-risk coupled nature of each financial institution. Indeed, each agent can act as generator or destroyer according to its financial risk position. With such a simple structure, a series of endogenous emergent properties arise in terms of financial stylized facts such as volatility cluster, excess volatility and heavy tails of the price returns distributions. Moreover, one can shed light on the bursty nature of boom-bust cycles in the financial 
market with consequent new insights on the onset of financial crisis and a new interpretation of the predictability of those crashes, since the time intervals between market collapses are power-law distributed as a consequence of the bursty nature of the system.

Another possible economic application of the model can be assessed in the field of international trade, where firm-to-firm connections represent the vast majority of world trade flows. Thoroughly explained by the authors in Bernard and Moxnes (2018) and Bernard et al. (2018), the literature on trade and production networks is still at an early stage, and there are a large number of unanswered empirical and theoretical questions. In particular, the dynamics of these interactions, e.g. why connections survive and how they evolve over time, are just beginning to be considered in this emerging literature. In recent years, thanks to the systematic digitization of customs, we have observed the emergence of big data on social and economic networks between firms and individuals. As a consequence, trade research has shifted attention to the role of individual firms, as an additional way to explore the large variation occurring in aggregate trade flows. Therefore, it is crucial to investigate the temporal structure of the firms' bilateral trading relationships and their evolution over time, in particular concerning the formation mechanism and duration of these importer-exporter relationships. The model presented in this paper fits very well with the sellers/buyers structure of trade, easily sketched as a bipartite network because a seller might link to a buyer, but a seller (buyer) cannot link to other sellers (buyers), taking into account that firms are typically sellers and buyers at the same time.

In terms of temporal network theory, the dynamics of firms' interactions can be investigated in terms of link fluctuations, and why connections survive and how they evolve over time. In looking at the nature of the connections between firms, an important issue has to do with assortativity among sellers and buyers. The ability of firms to create profitable and productive matches across borders is a key ingredient in aggregate trade flows and their growth. Therefore, it is valuable to explore the underlying sources of the frictions that prevent import-export matches, or cause them to be short-lived, which are still unknown.

\section{Conclusion}

In the present paper, we described the time evolution of the network model of XIE via a dynamics of coupled master equations. The model here investigated, due to its minimal dynamical rules, may shed light on a broad range of phenomena in complex systems with agents displaying opposed behaviors. Our analytic analysis correctly predicts both the transient and the equilibrium distributions of degree outside the transition, and our numerical analysis supports the existence of an extreme Thouless effect, i.e., a discontinuous jump and large dynamic fluctuations at the critical point where the sizes of extroverts and introverts populations coincide. From a more theoretical point of 
view, numerical simulations show that degree fluctuations in time are connected to a peak in degree correlation at the transition, suggesting the need to develop a theory beyond mean-field that will incorporate degree correlations to gain a better description at the transition. The Thouless effect predicts an explosive, non-linear, collective behavior at a precise critical point, suggesting that controlling for - exogenous or endogenous - changes in agent's behavior could keep the system far from abrupt transitions.

It is crucial to stress how the non-simultaneous asymmetric link formation criteria in the model gives birth to an intrinsically dynamic network with stationary states which change accordingly to the initial parameter of the network $h$ which is a measure of the heterogeneity among the nodes in the network. The maximal units' heterogeneity $h=0$ is the critical tipping point for which emergent properties arise related to the network connectivity as the anomalously large volatility and intense degree correlations can be further investigated for a better understanding the origin of bursts in financial dynamics.

In a broader perspective, the intermittency in the network, first introduced in this work, can give a more realistic explanation of the activated dynamics of complex systems, during which the system jumps from idle states to active ones, and vice-versa, giving to the entire system a dynamic long-range organization.

Finally, as a practical application in economics, we have shown how a simple combination of a non-simultaneous network structure with an adaptive agent based model spontaneously generates realistic financial dynamics which could have crucial policy implications. The bursty nature of the system, for certain market conditions, could be considered the seed of financial and economic fragility. Moreover, we have suggested some possible applications of the model - or similar generalizations - in the context of systemic risk and financial stability. On the other side, we underline the necessity to experimentally observe the existence of emergent structures in economic dynamics as, for example, in world trade economics, which could be assessed in terms of a temporal system which evolves in a non-simultaneous asymmetric picture as expressed in the network model presented in this paper.

\section{Acknowledgment}

This project has received funding from the European Union's Horizon 2020 research and innovation programme under grant agreement No 640772 - DOLFINS.

\section{Conflict of interest}

The authors declare that there are no conflicts of interest regarding the publication of this paper. 


\section{Appendix A Network degree distributions: a discussion}

Let us define a bipartite graph as as a triplet $G=(\top, \perp, E)$ where $\top$ is the set of high-target nodes (generator units) and $\perp$ is the set of low-target nodes (destroyer units), and $E \subseteq \top \times \perp$ is the set of links that can vary in the domain $[0,|\top| \times|\perp|]$ where $|\cdot|$ is the number of elements of the set.

Then, the time evolution of the this bipartite graph is an ordered sequence of $M$ graphs $\left\{G_{1}, G_{2}, \ldots, G_{M}\right\}$ defined over $N$ nodes, where each graph $G_{m}$ in the sequence represents the state of the network, i.e., the configuration of links in the time-window $\left[t_{m}, t_{m}+\Delta t_{m}\right], m=0, \ldots, M-1$.

The time-varying graph $G$ is fully described by means of a time-dependent adjacency matrix $A\left(t_{m}\right), m=0, \ldots, M-1$, where $a_{i j}\left(t_{m}\right)$ are the entries of the adjacency matrix of the graph at time $t_{m}$. Tuning the size of the time window used to build each snapshot, we obtain different representations of the system at different temporal scales.

In particular, in the limit case when $\Delta t \rightarrow 0$, we obtain an infinite sequence of graphs, where each graph corresponds to the configuration of connections at time instant $t$.

On the contrary if we set $\Delta t=T$, the time-varying graph degenerates into the corresponding aggregated graph, losing all the temporal information and obtaining a static graph.

In order to write an equation for the degree distributions of the graph we split the analytical description in terms of the two groups.

In principle we should know at each time step the configuration of the network $\mathcal{N}$ described for example by the adjacency matrix. We actually study the degree distribution defined as:

$$
\rho(k, t)=\sum_{\{\mathcal{N}\}} \sum_{i} \delta_{k k_{i}} P(\mathcal{N}, t) .
$$

where $\delta$ is the Kronecker delta, and $P(\mathcal{N}, t)$ is the probability to find our network in the configuration $\mathcal{N}$ at time $t$, where each node has degree $k_{i}$.

\section{A.1 Off-criticality: Bounded Poisson distribution}

Let us take into account that we know the correction factor of the cut rate $\epsilon$ in eq.(7) so we can define:

$$
\chi=\frac{\langle k\rangle_{0}}{\epsilon}
$$

In the stationary regime the master equation eq.(1) can be written:

$$
\left[\frac{k+1}{\chi} \rho_{1}^{s t}(k+1)-\rho_{1}^{s t}(k)\right]-\left[\frac{k}{\chi} \rho_{1}^{s t}(k)-\rho_{1}^{s t}(k-1)\right]=0
$$

with the boundary conditions as $\rho_{1}^{s t}(k=-1)=0$ and $\rho_{1}^{s t}\left(k=N_{0}+1\right)=0$. 
As proved by the authors in (Bassler et al., 2015), in such a closed system the stationary distribution corresponds to the steady state where the detailed balance relation is valid and fulfills the following recurrence equation:

$$
\rho_{1}^{s t}(k)=\frac{\chi}{k} \rho_{1}^{s t}(k-1)
$$

and by applying the recurrence successively we obtain the relation:

$$
\rho_{1}^{s t}(k)=\rho_{1}^{s t}(0) \prod_{m=1}^{k} \frac{\chi}{m} .
$$

Taking into account the normalization condition:

$$
\rho_{1}^{s t}(0)+\sum_{k=1}^{N_{0}} \rho_{1}^{s t}(k)=1,
$$

the stationary probability distribution in a finite network becomes:

$$
\rho_{1}^{s t}(k)= \begin{cases}\frac{\prod_{m=1}^{k} \frac{\chi}{m}}{1+\sum_{j=1}^{N_{0}} \prod_{m=1}^{j} \frac{\chi}{m}} & \text { for } k=1, \ldots, N_{0} \\ \frac{1}{1+\sum_{j=1}^{N_{0}} \prod_{m=1}^{j} \frac{\chi}{m}} & \text { for } k=0 .\end{cases}
$$

Since $\prod_{m=1}^{j} \frac{a}{m}=\frac{a^{j}}{j !}$, we get the the truncated poissonian distribution :

$$
\rho_{1}^{s t}(k)=\frac{1}{e^{\chi \frac{\Gamma\left(N_{0}+1, \chi\right)}{\Gamma(N 0+1)}-1}} \frac{\chi^{k}}{k !} \quad, k=0 \ldots N_{0}
$$

where $\Gamma\left(N_{0}+1, \chi\right)$ is the incomplete gamma function ${ }^{4}$. We replicated the simulations obtained by the authors in (Bassler et al., 2015) using a fixedpoint algorithm and we compared it to our numerical solutions. We have found that both approaches give identical numerical solutions as regards the degree distributions at equilibrium as shown in Fig.14 and the correspondent tables 1 and 2 where we also compute a statistical distance between distributions in terms of the Kullback-Leibler divergence (KL). This is a measure of how an

4 for $N_{0} \rightarrow \infty$ we can write $\sum_{j=1}^{N_{0}} \frac{a^{j}}{j !} \approx e^{a}-1$, yielding the Zero-truncated Poisson distribution:

$$
\rho_{1}^{s t}(k)=\frac{\chi^{k}}{\left(e^{\chi}-1\right)} \frac{1}{k !} \quad, k=0 \ldots N_{0}
$$


estimated probability distribution diverges from a true expected probability distribution estimated, in this case, from the network model. The results are that the CMED approach and the fixed-point algorithm provide the same solution in determining the stationary degree distributions. Despite the fact that the fixed-point approach converges faster to the solution, the CMED approach allows a complete description of the degree motion capturing also the transient states as well as being easy to modify to obtain more general agent-driven models. We observe that the information lost when we use the CMED and fixed-point distribution to approximate the real distribution is not zero even if very small. This is in line with the fact that we are neglecting the small correlations among the nodes in the network. Actually at criticality, the KL-divergence is much larger (typically two orders of magnitude), highlighting the presence of strong correlations that have been ignored in the present paper.

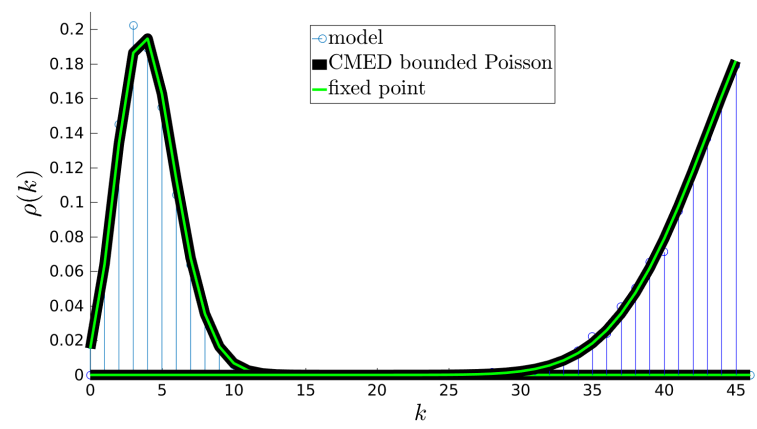

Fig. 14: Comparison among the three distributions obtained via Monte Carlo simulation of the model, using the CMED approach and then substituting the bounded poisson formula as in eq.(42) and then running the numerical solution through the fixed point algorithm used in (Bassler et al., 2015). We explore the case of $N=100$, the left distributions is for the case $N_{0}=55, N_{1}=45$ and the right distribution for $N_{0}=45, N_{1}=55$.

\begin{tabular}{llll}
\hline$N_{0}=45, N_{1}=55$ & Average Degree & Variance & KL divergence \\
\hline model (true distribution) & 41.525 & 10.224 & 0 \\
\cline { 1 - 3 } CMED Bounded Poisson & 41.582 & 10.001 & 0.0022275 \\
fixed point & 41.582 & 10.001 & 0.0022275 \\
\hline
\end{tabular}

Table 1

In order to have a full exploration of all the values of $h$ we need a consistent measure for the distance of the approximated distribution from the true one. We used the Hellinger distance since it is a metric satisfying the Cauchy-Schwarz inequality. In Fig.15, plotting the Hellinger distance versus 


\begin{tabular}{llll}
\hline$N_{0}=55, N_{1}=45$ & Average Degree & Variance & KL divergence \\
\hline model (true distribution) & 4.0878 & 4.1881 & 0 \\
\cline { 1 - 3 } CMED Bounded Poisson & 4.1772 & 4.1772 & 0.0023504 \\
fixed point & 4.1772 & 4.1772 & 0.0023504
\end{tabular}

Table 2

the population difference, we notice again that the approximation is pretty good for all the cases except for the critical point $h=0$ where the distance is 10 times larger.

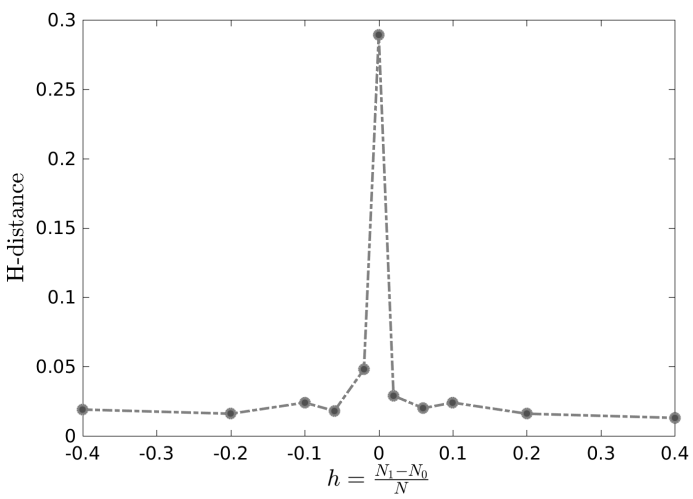

Fig. 15: Hellinger distance as measure of divergence between the estimated degree distribution (via CMED) and the true probability obtained from the model. We notice a peak at the critical value, again revealing a much stronger discrepancy between the two distributions since in our mathematical description we consider the network to be uncorrelated.

\section{A.2 Degree distribution at criticality}

In a radical view, the problem is symmetric in terms of the probability of cutting or adding a new link, so that approximately one can guess that $p_{0}^{(n)}(k) \sim$ $\rho_{1}(k)$. The meaning of this is that the probability of picking a destroyer which cuts a link with a generator of degree $k$ is the same as randomly picking a generator of degree $k$ and randomly cutting one of its links. Moreover at the critical point the symmetry is evident becasue $\rho_{1}(k)=\rho_{0}(q),\langle k\rangle=\langle q\rangle$ and there are almost no isolated nodes, so the detailed balance condition eq.(2) gives the relation for the conditional probabilities:

$$
q P(k \mid q)=k P(q \mid k)
$$

which is the symmetric relation for the conditional degree probabilities. 
We can build the connectivity matrix $\mathbf{C}=\left\{C_{q k}\right\}$ as defined by the author in (Boguná and Pastor-Satorras, 2002). The components of this matrix can be written as:

$$
C_{q k}=q P(k \mid q)
$$

which in the critical case is symmetric. These matrix elements measure the average number of links which, from a generator node of degree $k$, go to a destroyer node with degree $q$. For random bipartite networks, in which there are no correlations among the degrees of the nodes, we have that the connectivity matrix is given by $C_{q k}^{\mathrm{unc}}=q P(k \mid q)=q p_{0}^{(n)}(k)$.

In order to give an estimation of the degree distribution at criticality we try to recover a rough evaluation of the degree correlations, starting from the results of the monte-carlo simulations of the model.

In this case the neighbor probability can be generalized as $\tilde{p}_{0}^{(n)}(k)=$ $A_{(k, q)} p_{0}^{(n)}(k)$ where $A$ depends on both degrees of generators and destroyers. Notice that except in the uncorrelated case we have that $A=1$ for every pair $(k, q)$, so that the neighbor degree probability coincides with the conditional probability. We can imagine a raw approach for describing the degree distribution at criticality where the average degree correlation is of order 1, see Fig.8 and the assortativity is positively maximal, see Fig.10. If we define $\lambda:=A \frac{k}{\langle k\rangle}$, then at criticality the value $\lambda \approx 1$ except for the degrees near the boundaries $k=0$ and $k=N / 2$, which are the nodes for which the positive assortativity is lost and where we asymptotically recover the relation $\lambda=k /\langle k\rangle$.

With these conditions we can use the relation $\tilde{p}_{0}^{(n)}(k)=\lambda \rho_{1}(k)$, it is possible to write the master equation eq.(1) as :

$$
\left[\lambda \rho_{1}^{s t}(k+1)-\rho_{1}^{s t}(k)\right]-\left[\lambda \rho_{1}^{s t}(k)-\rho_{1}^{s t}(k-1)\right]=0
$$

with the reflecting boundary conditions $\rho_{1}^{s t}(k=-1)=0$ and $\rho_{1}^{s t}(k=$ $\left.N_{0}+1\right)=0$.

The recurrence equation $\lambda \rho_{1}^{s t}(k)-\rho_{1}^{s t}(k-1)=0$ has as solution:

$$
\rho_{1}(k)=C_{1}\left(\frac{1}{\lambda}\right)^{k-1}
$$

In the perfect symmetric case for $N \rightarrow \infty$ we have $\lambda=1$ so, recovering the constant $C_{1}$ from the normalization condition $\sum_{k=0}^{N / 2} \rho_{1}(k)=1$, we have:

$$
\rho_{1}^{s t}(k)=\frac{2}{N+2}
$$

which reminds one of the stationary distribution of a random walk inside reflecting barriers.

The stationary distribution eq.(47) is correct as far as the dynamics is not affected by the two barriers, and it happens only in the thermodynamic limit where $N \rightarrow \infty$. For small networks the barriers' effects are intense and the distribution appears to be closer to a platykurtic bell-shape curve rather then a uniform one. At the moment we are not able to write an analytical 
expression of the correlations $\lambda$ for the varius degree $k$. In order to give a rough estimation of the platykurtic distribution at criticality, we will make strong approximations creating a piecewise function for the correlation factor $\lambda$. We define three regions that take into account the fact that the average correlation is of order $\rho \lesssim 1$. We consider the degrees $k$ close to the boundaries to be of the same order as in the uncorrelated case, i.e., $k /\langle k\rangle$. Then we choose the intermediate values of $k$ with degree correlation 1 . Studying the assortative structure of the aggregate ANND it is possible to estimate the $k_{\min }$ and $k_{\max }$ for which the degree correlations are of order 1, see Fig.16.

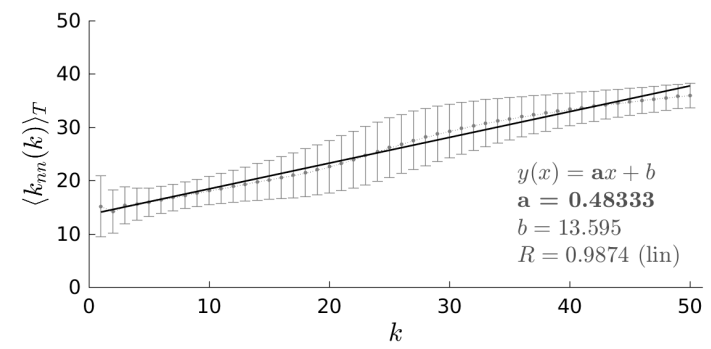

(a) Linear regression for the aggregate ANND for $N=$ 100 at criticality and a strength of assortativity of $a \approx$ 0.5 . Here we estimated $k_{\min }=13$ and $k_{\max }=37$.

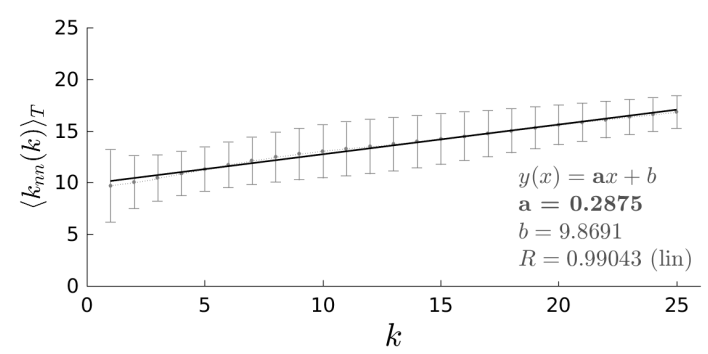

(b) Linear regression for aggregate ANND for $N=50$ at criticality and a strength of assortativity of $a \approx 0.3$.

Fig. 16: Study of the strength of the aggregate assortativity $\left\langle k_{n n}(k)\right\rangle_{T}=a k$ for which increasing the size of the network we have $a \rightarrow 1$, that is the condition of perfect assortativity. From such study it is also possible define the $k_{\min }$ and $k_{\max }$ as the minimum and maximum value of the $\left\langle k_{n n}(k)\right\rangle_{T}$.

In such a way the overall correlation of the system is $\rho$, and a particular choice of the $\lambda$ dependence can be made as;

$$
\lambda=\left\{\begin{array}{l}
\lambda_{1}=\frac{k_{\min }}{N / 4} \quad, k \in\left[0, k_{\min }\right] \\
\lambda_{2}=1 \quad, k \in\left(k_{\min }, k_{\max }\right) \\
\lambda_{3}=\frac{k_{\max }}{N / 4} \quad, k \in\left[k_{\max }, N / 2\right]
\end{array}\right.
$$


where the width of the interval of maximal corrleation $\left(k_{\min }, k_{\max }\right)$ is evaluated as $\rho N / 2$. At this point we can evaluate the solution eq. (46) for the three regions and using the continuity requirement and the normalization condition, we get:

$$
\left\{\begin{array}{l}
C_{2}^{-1}=\frac{\lambda_{1}^{k \min }}{\lambda_{2}^{k \min }} \sum_{k=1}^{k_{\min }}\left(\frac{1}{\lambda_{1}}\right)^{k}+\sum_{k=k_{\min }+1}^{k_{\max }-1}\left(\frac{1}{\lambda_{2}}\right)^{k}+\frac{\lambda_{3}^{k \max }}{\lambda_{2}^{k m a x}} \sum_{k=k_{\min }}^{N / 2}\left(\frac{1}{\lambda_{3}}\right)^{k} \\
C_{1}=C_{2} \frac{\lambda_{1}^{k \min }}{\lambda_{2}^{k \min }} \\
C_{3}=C_{2} \frac{\lambda_{3}^{k \max }}{\lambda_{2}^{k \max }}
\end{array}\right.
$$

Using those conditions we get a degree distribution as shown in Fig.17 similar to that of a random walk process under "soft" boundary conditions (Liu et al., 2013) rather then the reflecting barriers condition which instead brings one to a uniform distribution. We also compare the real model distribution with the poisson distribution under the uncorrelated hypothesis. One can notice how our numerical evaluation replicates better the behavior of the real distribution.

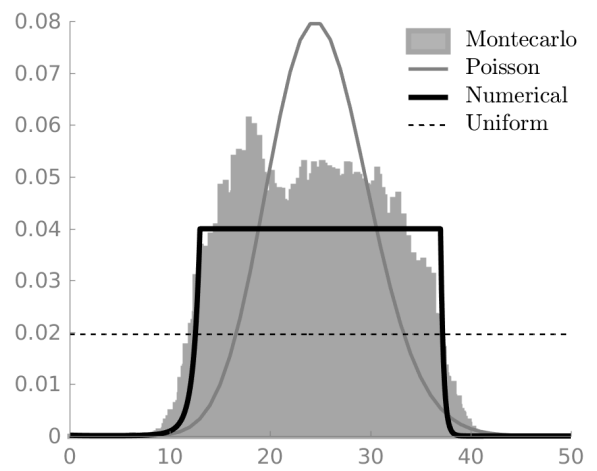

Fig. 17: Degree distribution $\rho_{1}(k)$ for the critical case $N_{1}=N_{0}=N / 2$. In the case of $N=100$ the average degree correlation is $\rho \approx 0.8$ and $k_{\min }=13, k_{\max }=37$, so that the numerical distribution (bold black line) has been reconstructed as a piecewise distribution. So our distribution has the correct mean and also the right variance with respect to the uncorrelated distribution (Poisson) and with respect to the thermodynamic $\operatorname{limit}(N \rightarrow \infty)$ of a uniform distribution.

The exact analytical functional of $\lambda$ would give the possibility to simulate the network to get the exact distribution which at this point in the study has not been possible to find.

It is interesting to notice that our numerical estimation requires three parameters to find an approximated distribution for the correlated case of criticality. On the contrary in the uncorrelated case a distribution with only one parameter is enough to have the system described. As a further check of this 


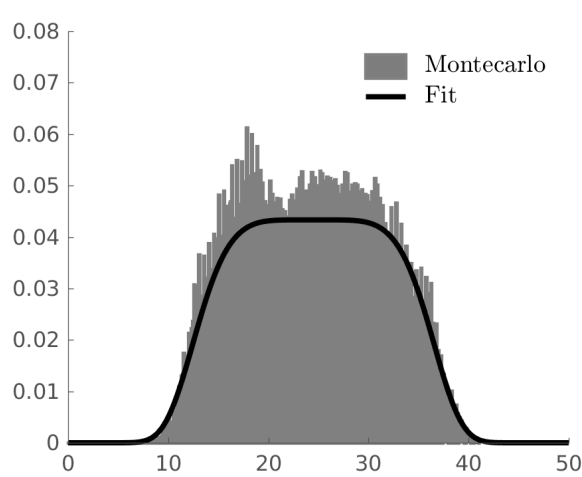

Fig. 18: Fit of the degree distribution of the network at criticality $(N=100)$ and fitting curve (black line) under the assumption of a generalized normal distribution (also called an exponential power distribution). Parameter estimation via maximum likelihood gives an average of $\mu=24.89$, a shape parameter of $\beta=5.27$ and the scale parameter $\sigma=9.14$ (The estimation has been addressed using the statistical tool in (Mineo et al., 2005)). The results are in line with the mean of the distribution and its variance.

view we estimated the montecarlo distribution with a three-parameters fit using a generalized normal distribution $p^{*}(k) \propto \exp \left\{-\frac{|k-\mu|^{\beta}}{\beta \sigma^{\beta}}\right\}$. We find remarkably good fit results shown in Fig.18, so supporting the necessity of three parameters to fully characterize the degree distribution of the network at criticality where we have strong correlations and the mean field approximation fails. 


\section{References}

Albert, R. and Barabási, A.-L. (2002). Statistical mechanics of complex networks. Reviews of modern physics, 74(1):47.

Bardoscia, M., De Luca, G., Livan, G., Marsili, M., and Tessone, C. J. (2013). The social climbing game. Journal of Statistical Physics, 151(3-4):440-457.

Barucca, P. and Lillo, F. (2015). The organization of the interbank network and how ecb unconventional measures affected the e-mid overnight market. arXiv preprint arXiv:1511.08068.

Bassler, K., Liu, W., Schmittmann, B., and Zia, R. (2015). Extreme thouless effect in a minimal model of dynamic social networks. Physical Review E, 91(4):042102.

Battiston, S., Farmer, J. D., Flache, A., Garlaschelli, D., Haldane, A. G., Heesterbeek, H., Hommes, C., Jaeger, C., May, R., and Scheffer, M. (2016). Complexity theory and financial regulation. Science, 351(6275):818-819.

Bernard, A. B., Bøler, E. A., and Dhingra, S. (2018). Firm-to-firm connections in colombian imports. Technical report, National Bureau of Economic Research.

Bernard, A. B. and Moxnes, A. (2018). Networks and trade. Annual Review of Economics, (0).

Boguná, M. and Pastor-Satorras, R. (2002). Epidemic spreading in correlated complex networks. Physical Review E, 66(4):047104.

Caldarelli, G., Capocci, A., De Los Rios, P., and Munoz, M. A. (2002). Scalefree networks from varying vertex intrinsic fitness. Physical review letters, $89(25): 258702$.

Dorogovtsev, S. N. and Mendes, J. F. (2013). Evolution of networks: From biological nets to the Internet and $W W W$. OUP Oxford.

Fricke, D. (2012). Trading strategies in the overnight money market: Correlations and clustering on the e-mid trading platform. Physica A: Statistical Mechanics and its Applications, 391(24):6528-6542.

Gardiner, C. (2010). Stochastic Methods : A Handbook for the Natural and Social Sciences. Springer Berlin, Berlin.

Goh, K.-I. and Barabási, A.-L. (2008). Burstiness and memory in complex systems. EPL (Europhysics Letters), 81(4):48002.

Goyal, S. (2012). Connections: an introduction to the economics of networks. Princeton University Press.

Grandjean, M. (2016). A social network analysis of twitter: Mapping the digital humanities community. Cogent Arts \& Humanities, 3(1):1171458.

Holme, P. and Saramäki, J. (2012). Temporal networks. Physics reports, $519(3): 97-125$.

Holme, P. and Saramäki, J. (2013). Temporal networks. Springer.

Iori, G., De Masi, G., Precup, O. V., Gabbi, G., and Caldarelli, G. (2008). A network analysis of the italian overnight money market. Journal of Economic Dynamics and Control, 32(1):259-278.

Iori, G., Reno, R., De Masi, G., and Caldarelli, G. (2007). Trading strategies in the italian interbank market. Physica A: Statistical Mechanics and its 
Applications, 376:467-479.

Jackson, M. O. (2010). Social and economic networks. Princeton university press.

Jackson, M. O. and Wolinsky, A. (1996). A strategic model of social and economic networks. Journal of economic theory, 71(1):44-74.

Karsai, M., Jo, H.-H., and Kaski, K. (2018). Bursty human dynamics. Springer.

Kitsak, M. and Krioukov, D. (2011). Hidden variables in bipartite networks. Physical Review E, 84(2):026114.

Kondor, I., Csabai, I., Papp, G., Mones, E., Czimbalmos, G., and Sándor, M. C. (2014). Strong random correlations in networks of heterogeneous agents. Journal of Economic Interaction and Coordination, 9(2):203-232.

Lambert, D. and Vanni, F. (2018). Complexity and heterogeneity in a dynamic network. Chaos, Solitons \& Fractals, 108:94-103.

Liu, W., Schmittmann, B., and Zia, R. (2013). Extraordinary variability and sharp transitions in a maximally frustrated dynamic network. EPL (Europhysics Letters), 100(6):66007.

Masuda, N. and Lambiotte, R. (2016). A guide to temporal networks, volume 4. World Scientific.

Mineo, A. M., Ruggieri, M., et al. (2005). A software tool for the exponential power distribution: The normalp package. Journal of Statistical Software, $12(4): 1-24$.

Newman, M. (2010). Networks: an introduction. Oxford university press.

Pastor-Satorras, R., Vázquez, A., and Vespignani, A. (2001). Dynamical and correlation properties of the internet. Physical review letters, 87(25):258701.

Perra, N., Gonçalves, B., Pastor-Satorras, R., and Vespignani, A. (2012). Activity driven modeling of time varying networks. Scientific reports, 2.

Saracco, F., Di Clemente, R., Gabrielli, A., and Squartini, T. (2015). Randomizing bipartite networks: the case of the world trade web. Scientific reports, 5.

Ubaldi, E., Perra, N., Karsai, M., Vezzani, A., Burioni, R., and Vespignani, A. (2016). Asymptotic theory of time-varying social networks with heterogeneous activity and tie allocation. Scientific reports, 6 .

Vanni, F. and Barucca, P. (2017). Time evolution of an agent-driven network model. Technical report, LEM working paper series, Sant'Anna School of Advanced Studies, Pisa, Italy.

Vanni, F., Napoletano, M., Roventini, A., and Dosi, G. (2018). An Endogenous money network model of leveraged institutions. Paper-Progress.

Vázquez, A., Pastor-Satorras, R., and Vespignani, A. (2002). Large-scale topological and dynamical properties of the internet. Physical Review E, 65(6):066130. 